\title{
Order Tracking Using Variational Mode Decomposition to Detect Gear Faults under Speed Fluctuations
}

\author{
Madhurjya D. Choudhury ${ }^{1}$, Liu Hong ${ }^{2}$, and Jaspreet S. Dhupia ${ }^{3}$ \\ ${ }^{1,3}$ University of Auckland, Auckland, 1010, New Zealand \\ mcho491@aucklanduni.ac.nz \\ j.dhupia@auckland.ac.nz. \\ ${ }^{2}$ Wuhan University of Technology, Wuhan, P. R. China \\ hongliu@whut.edu.cn
}

\begin{abstract}
Fault detection in gearboxes plays a significant role in ensuring their reliability. Vibration signals collected during gearbox operation contain a wealth of valuable condition information that can be exploited for fault detection. However, in an industrial environment machine operating speed always fluctuates around its nominal value, which causes smearing of the gearbox vibration spectrum. Considering operating speed fluctuation and multicomponent nature of measured gearbox vibration signals, an order-tracking method combining the variational mode decomposition (VMD) and the fast dynamic time warping (FDTW) is proposed in this paper. Firstly, the multicomponent vibration signal is decomposed into several intrinsic mode functions (IMFs) using VMD in order to extract a signal component with higher signal-to-noise ratio (SNR). Then, the sensitive fault information carrying IMF is exploited to estimate the instantaneous speed profile in order to construct the shaft rotational vibration signal. The measured vibration signal is then resampled based on the optimal warping path obtained by FDTW, which performs an "elastic" stretching and compression along the time axis of the extracted shaft vibration signal with respect to a sinusoidal reference signal of constant shaft rotational frequency. Finally, the gear fault is detected by constructing the order spectrum of the resampled vibration signal. The effectiveness of the proposed algorithm is demonstrated using simulation results.
\end{abstract}

\section{INTRODUCTION}

The presence of localized defects in a gear tooth results in periodic amplitude and phase modulations in the measured

Madhurjya Choudhury et al. This is an open-access article distributed under the terms of the Creative Commons Attribution 3.0 United States License, which permits unrestricted use, distribution, and reproduction in any medium, provided the original author and source are credited. vibration signal (Randall, 1982). This phenomenon introduces sidebands around the gear-mesh frequency (GMF) and its harmonics, in the vibration signal spectrum. Detecting these sidebands, which occur at the fault characteristic frequencies (FCFs), is the basis of identifying gear faults. However, extracting fault information from them is challenging because the FCFs and GMF are related to the shaft rotating frequency, which varies during operation (Wang, Cheng, Qiao \& Qu, 2018). This operating speed fluctuation results in a non-stationary nature of the measured vibration signal. Under such circumstances, the traditional Fourier transform based spectral analysis fails to provide reliable fault information as frequency overlapping occurs leading to the undesirable smearing phenomenon of the frequency components (Wang et al., 2018). Moreover, the measured vibration signals are generally multi-component in nature and often contaminated by unwanted background noise. Thus, developing appropriate signal processing techniques that can successfully extract relevant faultinformation carrying part from the measured signal and remove the influence of the operating speed fluctuation to extract the fault characteristics is an important research aspect in the field of gear fault diagnostics.

Traditionally, order-tracking (OT) is adopted to remove spectral smearing, which transforms a non-stationary signal in the time domain into a stationary one in the angular domain (Schmidt, Heyns \& deVilliers, 2018). OT requires collection of extra information on the shaft rotational speed or angular position using additional sensors like tachometers and encoders (Bonnardot, El Badaoui, Randall, Daniere \& Guillet, 2005). However, the availability of such sensors in an industrial set-up is limited due to the added cost associated with it. Hong, Qu, Dhupia, Sheng, Tan, and Zhou (2017) proposed a fast dynamic time warping (FDTW) based tacholess order tracking technique where spectral smearing was alleviated by aligning a filtered shaft rotational harmonic with a sinusoidal reference signal constructed assuming 
constant shaft speed. A critical step in this method, which requires user intervention and understanding of system operation, is the extraction of the mono-component shaft rotational harmonics using band-pass filtering, from a multicomponent measured vibration signal. However, the central frequency and bandwidth of this filter need to be chosen carefully, especially when speed fluctuations are involved (Cheng, Yu, Tang \& Yang, 2008). If these parameters are not estimated properly, the resultant signal may lead to inaccurate interpretations, due to the varying frequency content of measurements. As a result, researchers have recently started adopting more adaptive approaches using different signal decomposition methods, like the variational mode decomposition (VMD), which uses advanced filtering techniques to extract signal modes that can adaptively update the center frequency and bandwidth until the appropriate mode is identified (Dragomiretskiy \& Zosso, 2014; Feng, Zhang \& Zuo, 2017). VMD is a non-recursive decomposition scheme, which solves a constrained variational problem to concurrently extract the signal modes (Dragomiretskiy \& Zosso, 2014).

In this paper, a novel tacho-less order tracking method for self-adaptive gear fault-detection is developed by combining VMD and FDTW. The detection algorithm first employs VMD to extract the time-varying gear-mesh frequency component mode from the vibration signal of a gearbox. Afterwards, the extracted component is exploited to estimate the instantaneous speed profile in order to construct the shaft rotational vibration signal. The extracted component is then utilized to resample the measured original vibration signal of the gearbox based on an optimal warping path evaluated from the FDTW. The spectrum of the original vibration signal after the resampling step is found to improve as the smeared gearmesh frequency and the fault-induced sidebands are easily identifiable as compared to the spectrum in the presence of speed fluctuation and noise. The capability of the proposed algorithm is demonstrated using an analytical vibration signal model of a fixed-axis gearbox.

The rest of the paper is organized as follows. Section 2 gives a brief background of VMD and FDTW followed by the introduction of the proposed algorithm. Section 3 investigates the effectiveness of the proposed algorithm using a MATLAB-based simulation analysis. Finally, the paper is concluded in Section 4.

\section{PRINCIPLE OF THE PROPOSED FAULT DETECTION ALGORITHM}

The proposed algorithm sequentially uses VMD and FDTW on measured vibration signal to perform fault detection in gears operating under variable speed. Accordingly, a brief background on VMD and FDTW is first provided in this section for the ease of understanding of the readers. The proposed fault detection algorithm is also introduced later in this section.

\subsection{Variational mode decomposition (VMD)}

VMD is an adaptive decomposition method that can decompose a complex multi-component signal $x(t)$ into a discrete number of modes or IMFs, $m_{k}(t)$. Here, $m_{k}(t)$ is the $k$ th mode or IMF, which is defined as an amplitude-frequency modulated (AM-FM) signal and can be written as (Dragomiretskiy \& Zosso, 2014),

$$
m_{k}(t)=A_{k}(t) \cos \left(\phi_{k}(t)\right),
$$

where $A_{k}(t)$ is the instantaneous amplitude, $\phi_{k}(t)$ is the instantaneous phase whose derivative gives the instantaneous frequency.

VMD produces IMFs, which are signals with limited bandwidth around their central frequencies. During the decomposition process, VMD iteratively updates each IMF $m_{k}(t)$ in the frequency domain, simultaneously estimating their associated center frequency $\omega_{k}$ as the center of gravity of the respective IMF's power spectrum. In order to estimate the bandwidth of a particular IMF $m_{k}(t)$, its analytic signal is constructed by applying Hilbert transform in order to calculate the unilateral non-negative frequency spectrum. Then the IMF's spectrum is shifted to baseband by multiplying it with an exponential function tuned to its estimated center frequency. Finally, the required bandwidth is estimated using the $H^{1}$ Gaussian smoothness. The resulting constrained variational problem, with the goal to minimizing the sum of the spectral widths of all the IMFs, is as follows (Dragomiretskiy \& Zosso, 2014; Feng et al., 2017),

$$
\begin{aligned}
& \min _{\left\{m_{k}\right\},\left\{\omega_{k}\right\}}\left\{\sum_{k=1}^{K}\left\|\partial_{t}\left[\left(\delta(t)+\frac{j}{\pi t}\right) * m_{k}(t)\right] e^{-j \omega_{k} t}\right\|_{2}^{2}\right\} \\
& \text { s.t. } \sum_{k=1}^{K} m_{k}=x(t),
\end{aligned}
$$

where $\quad\left\{m_{k}\right\}=\left\{m_{1}, \ldots, m_{K}\right\} \quad$ and $\quad\left\{\omega_{k}\right\}=\left\{\omega_{1}, \ldots, \omega_{K}\right\} \quad$ are, respectively, the mode vector and their corresponding center frequency vector, $\delta($.$) is the Dirac delta function, * denotes$ the convolution function, $K$ is the number of modes or IMFs to be extracted, and the constraint denotes that the sum of the modes is equal to the original signal $x(t)$.

The constrained optimization problem in Eq. (2) is solved by converting it into an unconstrained problem by introducing a quadratic penalty term and Lagrangian multiplier as,

$$
\begin{aligned}
& L\left[\left\{m_{k}\right\},\left\{\omega_{k}\right\}, \lambda\right]=\alpha \sum_{k=1}^{K}\left\|\partial_{t}\left[\left(\delta(t)+\frac{j}{\pi t}\right) * m_{k}(t)\right] e^{-j \omega_{k} t}\right\|_{2}^{2} \ldots \\
& +\left\|x(t)-\sum_{k=1}^{K} m_{k}(t)\right\|_{2}^{2}+\left\langle\lambda(t), x(t)-\sum_{k=1}^{K} m_{k}(t)\right\rangle,
\end{aligned}
$$

where $\alpha$ is the penalty term and $\lambda(t)$ is the Lagrangian multiplier. Now the solution to the original minimization problem of Eq. (2) is found by iteratively solving the Lagrangian $L$ of Eq. (3) using alternate direction method of 
multipliers (ADMM), which is explained in detail in (Dragomiretskiy \& Zosso, 2014). Next, the IMF $m_{k}(t)$ and its center frequency $\omega_{k}$ is obtained by updating them as a solution to a minimization problem of Eq. (3) as (Feng et al., 2017),

$$
\begin{gathered}
m_{k}(t)=\underset{m_{k}}{\arg \min } L\left[\left\{m_{k}\right\},\left\{\omega_{k}\right\}, \lambda\right], \\
\omega_{k}=\underset{\omega_{k}}{\arg \min } L\left[\left\{m_{k}\right\},\left\{\omega_{k}\right\}, \lambda\right] .
\end{gathered}
$$

In the frequency domain, the solution to Eq. (4) can be found as (Feng et al., 2017),

$$
\hat{m}_{k}(\omega)=\frac{\hat{x}(\omega)-\sum_{i \neq k} \hat{m}_{i}(\omega)+\frac{1}{2} \hat{\lambda}(\omega)}{1+2 \alpha\left(\omega-\omega_{k}\right)^{2}} .
$$

The full spectrum of the IMF is then obtained by applying Hermitian symmetric completion and it is converted into the time domain by taking the real part of the inverse Fourier transform of the filtered analytic signal.

As previously mentioned, the center of gravity of the obtained IMF $m_{k}(t)$ power spectrum is utilized to calculate its center frequency $\omega_{k}$ as (Feng et al., 2017),

$$
\omega_{k}=\frac{\int_{0}^{\infty} \omega\left|\hat{m}_{k}(\omega)\right|^{2} d \omega}{\int_{0}^{\infty}\left|\hat{m}_{k}(\omega)\right|^{2} d \omega} .
$$

The detailed algorithm of VMD is provided in (Dragomiretskiy \& Zosso, 2014) and its MATLAB code is available at (Zosso, 2013). The readers are advised to refer these for more details on this method.

\subsection{Fast dynamic time warping (FDTW)}

FDTW is an approximate method for multilevel implementation of dynamic time warping (DTW) approach with a linear $\mathrm{O}(N)$ time and space complexity which produces an accurate minimum-distance warp path between two time series that is nearly optimal (Salvador \& Chan, 2007).

DTW is an approach to achieve an optimal alignment between two time series in order to determine the similarity between the two given series. The resemblance between the given time series is estimated by optimal alignment between their corresponding data points, which yields a minimum residue, allowing the two timescales to be warped nonlinearly by stretching or shrinking (Salvador \& Chan, 2007; Hong et al., 2017). In order to understand the concept of DTW, two time series, $X\left(=x_{1}, x_{2}, \ldots, x_{i}, \ldots, x_{N}\right)$ of length $N$ and $Y\left(=y_{1}\right.$, $\left.y_{2}, \ldots, y_{i}, \ldots, y_{M}\right)$ of length $M$, are considered. Next a warping path, $W\left(=w_{1}, w_{2}, \ldots, w_{k}, \ldots, w_{P}\right)$ is constructed, where $P$ is the length of the warping path and the $k$-th element of the warping path is given by,

$$
w_{k}=(i, j)
$$

where $i\left(=w_{k}(1,1)\right)$ is an index from the time series $X$, and $j$ $\left(=w_{k}(1,2)\right)$ is an index from the time series $Y$. Now, if the warping path, $W$, contains the element, $(i, j)$ then it implies that DTW has performed an alignment between the $i$-th sample of $X$ and the $j$-th sample of $Y$. The warping path must also satisfy the following conditions (Salvador \& Chan, 2007),

1. The first element of $W$ must be $w_{l}=(1,1)$, which implies that the warping path must start at the beginning of each time series,

2. The last element of $W$ must be $w_{P}=(N, M)$, which implies that the warping path must finish at the end of both the time series, and

3. Moreover, every index in both of the time series, between the start and end of the warping path, must be used sequentially at least once, i.e., if $w_{k}=(i, j)$ and $w_{k+1}$ $=\left(i^{\prime}, j^{\prime}\right)$, then $i^{\prime} \in(i, i+1)$ and $j^{\prime} \in(j, j+1)$.

The warping path $W$ is evaluated by calculating a twodimensional cost matrix, $\mathbf{D}$, of dimension, $N \times M$, as shown in Fig. 1. Each cell of the matrix $\mathbf{D}$ is calculated as,

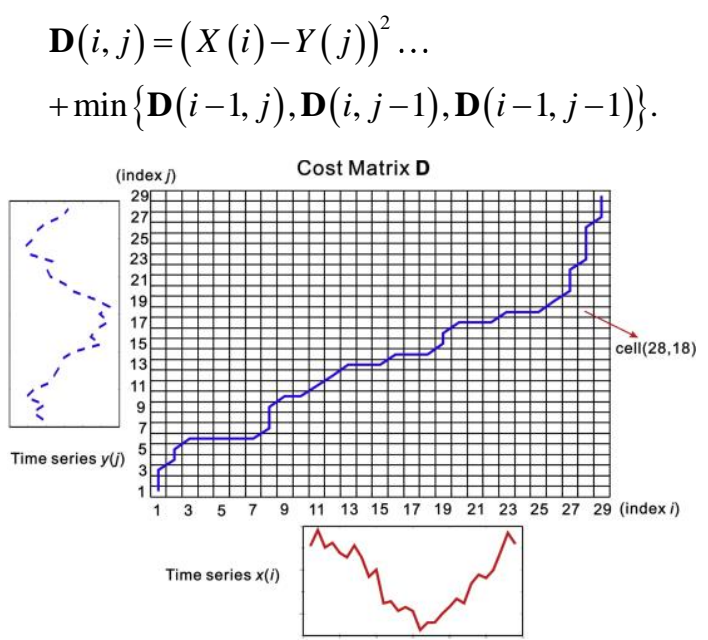

Figure 1. The two-dimensional cost matrix, D, of DTW indicating the optimal alignment path for the time series, $X$ and $Y$ (Hong et al., 2017).

As each cell in the cost matrix D is evaluated once, so DTW becomes quadratic in time and space and it creates the need for methods to speed up the algorithm. FDTW is the faster alternative of DTW, which is achieved by a multilevel recursive approach with three key operations (Salvador \& Chan, 2007; Hong et al., 2017):

1. Coarsening: In this step, a lower resolution time series is created by considering half as many points as the input time series for a single run of the recursive function. Coarsening is run several times until a lowest resolution of the time series is obtained, which is determined by a pre-defined radius.

2. Projection: This step involves finding an optimal warping path in the lowest resolution using the standard 
DTW and then project it to the next higher resolution, which is obtained by increasing the lower resolution by a factor of 2 .

3. Refinement: In this step, the projected path is expanded by the predefined radius to form a search window at a higher resolution, which is passed to a constrained DTW algorithm. This constrained DTW only evaluates the cells within the search window to find the optimal warping path.

Thus, in FDTW, the DTW algorithm only evaluates the cells within the search window rather than the complete cost matrix, which speeds up the overall algorithm.

\subsection{Proposed fault detection algorithm}

The proposed fault detection algorithm is developed to first extract a mode with a high signal-to-noise ratio (SNR) from the measured vibration signal and then use it to counter the spectral smearing phenomenon. The key steps of the developed fault detection scheme are:

Step 1: Decomposition of the measured vibration signal into different signal components using VMD in order to extract the fault information carrying part of the measured signal.

Step 2: Estimation of the shaft vibration signal from the selected signal mode.

Step 3: Estimation of a reference signal with an assumed constant speed.

Step 4: FDTW implementation to align the extracted shaft vibration signal with the reference signal.

Step 5: Reconstruction and resampling of the original vibration signal to alleviate the spectral smearing caused by speed fluctuations.

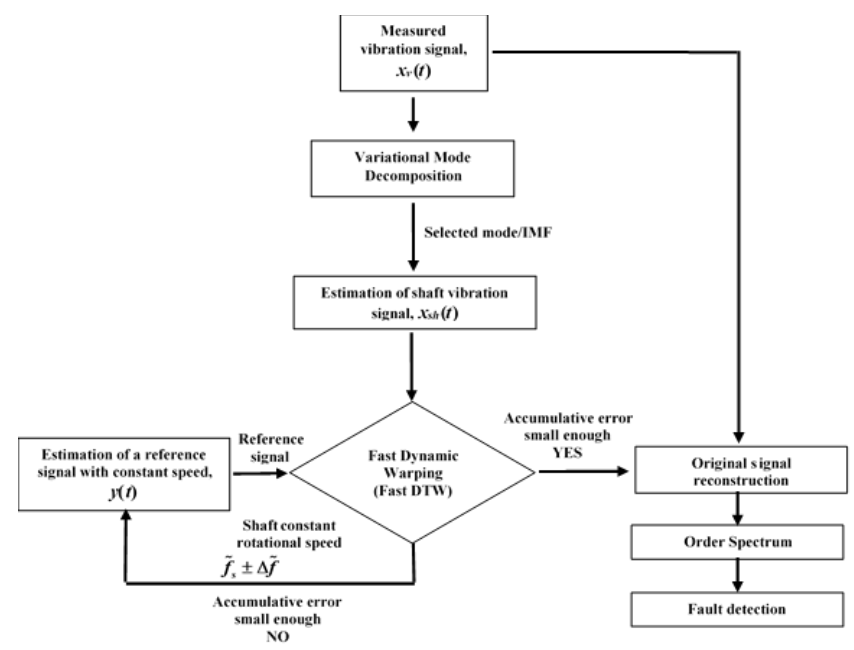

Figure 2. Flowchart of the proposed fault detection algorithm.
The overall scheme is summarized in Fig. 2 and is explained in detail using a MATLAB-based simulation of a fixed-axis gearbox in Section 3.

\section{SimUlation BASED ANALYSIS}

This section describes the model to represent the vibration signal of a single stage fixed-axis gearbox (with number of teeth on pinion $N_{p}=10$ and number of teeth on gear $N_{g}=15$ ) operating under speed fluctuation. The signal obtained from this model is processed by the proposed fault detection algorithm to study the capability of the method in extracting fault indicators under variable speed conditions.

The vibration response, $x_{v}(t)$, of the virtual gearbox consists of the shaft-vibration signal, $x_{s}(t)$, and gear-mesh vibration signal, $x_{m}(t)$. The dominant frequency components of $x_{s}$ are the gear-shaft rotational frequency, $f_{s}(t)$. The important frequency components in $x_{m}$ are the fundamental gear-mesh frequency, $f_{m}(t)\left(=N_{g} f_{s}(t)\right)$, and the sidebands around the mesh frequency if a local gear fault is present. Thus, the overall vibration signal, $x_{v}(\mathrm{t})$, can be simulated as (Hong et al., 2017; McFadden, 1986),

$$
\begin{aligned}
& x_{v}(t)=x_{s}(t)+x_{m}(t) \\
& =\sum_{n=1}^{N} A_{s n}(t) \cos \left(2 \pi n f_{s}(t) t+\theta_{n}\right) \ldots \\
& +\sum_{m=1}^{M} A_{m}\left(1+a_{m}(t)\right) \cos \left(2 \pi m f_{m}(t) t+b_{m}(t)+\varphi_{m}\right),
\end{aligned}
$$

where a speed variation function, $f_{s}(t)=(1+0.05 t) f_{s}$, is used to simulate a speed fluctuation around the nominal shaft rotational frequency $f_{s}=20 \mathrm{~Hz}$. Fig. 3 plots the speed profile, $f_{s}(t)$. The amplitude and phase modulation (AM \& PM) functions, $a_{m}(\mathrm{t})$ and $b_{m}(t)$, due to a local fault at a gear tooth are expressed as,

$$
\begin{aligned}
& a_{m}(t)=\sum_{q=1}^{Q} a_{m q} \cos \left(2 \pi q f_{s}(t) t+\delta_{m q}\right) \\
& b_{m}(t)=\sum_{q=1}^{Q} b_{m q} \cos \left(2 \pi q f_{s}(t) t+\beta_{m q}\right) .
\end{aligned}
$$

If the gear is free from any defect, then the amplitudes of Eq. (11) and Eq. (12) are set equal to 0.

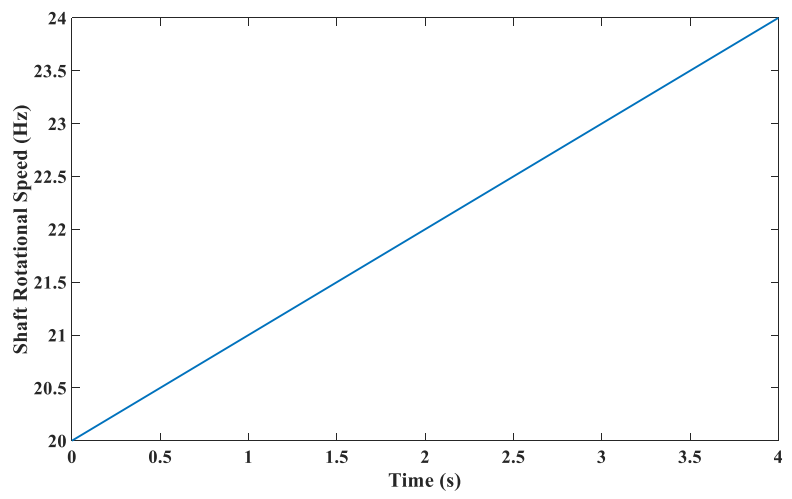

Figure 3. Speed fluctuation profile used in the simulation. 


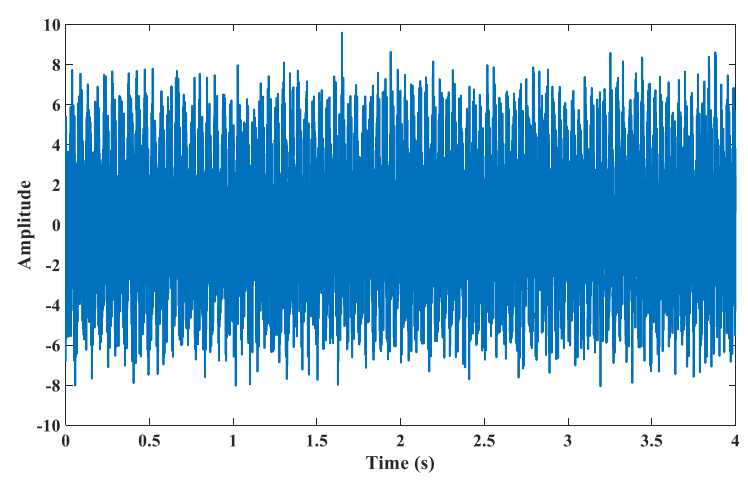

Figure 4. Time domain signal of the simulated faulty gearbox.

Without the loss of generality, the first gear mesh harmonic ( $m=1)$ is considered and its corresponding amplitude is taken as $A_{l}=3$. The harmonic order $(q)$ of the modulation functions $a_{m}(\mathrm{t})$ and $b_{m}(t)$, is assumed to be 2 , with their corresponding amplitudes set as $a_{11}=0.15, a_{12}=0.30, b_{11}=0.18, b_{12}=0.25$. For the gear shaft, only the fundamental harmonics is considered (i.e., $n=1$ ) and its corresponding amplitude $A_{\mathrm{s} 1}$ is chosen as 2. A Gaussian white noise of $\mathrm{SNR}=5 \mathrm{~dB}$ is added to the vibration signal $x_{v}(t)$ to account for the challenges encountered in practical measurements. Fig. 4 shows the time-domain waveform of the simulated gearbox, which indicates that the modulation features caused by the gear faults are mostly suppressed by noise and other unwanted components. This study is done considering a sampling frequency of $4096 \mathrm{~Hz}$.

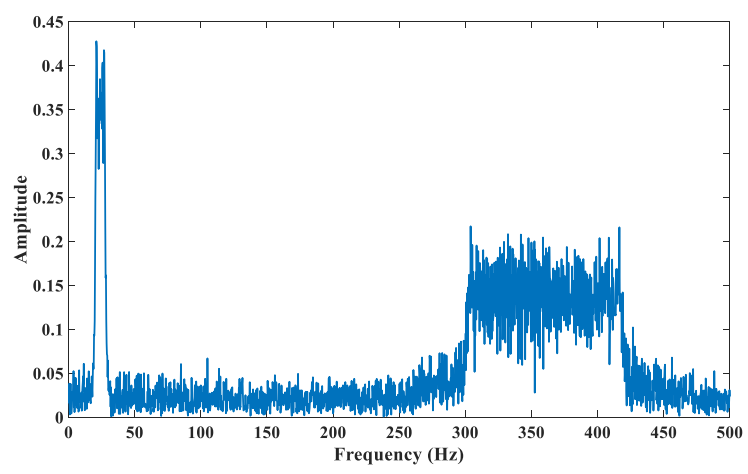

Figure 5. The spectrum of the raw vibration signal of the simulated gearbox in fault condition in the presence of speed fluctuation and noise.

The spectrum of the simulated signal is shown in Fig. 5. However, the presence of sidebands around the meshing harmonics due to gear faults cannot be distinguished from the spectral components because of the smearing phenomenon caused by shaft operating speed fluctuation. Therefore, in order improve the smeared spectra; the proposed algorithm is applied, which is explained in detail in the subsequent section.

\subsection{Application of the proposed fault detection algorithm}

In this section, the detection algorithm is applied on the simulation model and an explanation of the involved steps is provided.

\subsubsection{Step 1: Signal decomposition using VMD}

In this step, VMD is applied on the simulated vibration signal $x_{v}(t)$ in order to decompose it into its constituent modes. Fig. 6 shows the IMFs generated after applying VMD with initial mode estimation of $K=5$. VMD is found to be effective in decomposing the multi-component vibration signal $x_{v}(t)$ into its constituent modes. After the decomposition, IMF 2 is found to have an instantaneous frequency (IF) centering about the gear-mesh frequency and so it is selected for further analysis. Fig. 7 shows the IF profile of IMF 2. Since the mesh frequency is a function of the shaft rotational frequency, it can be exploited to estimate the instantaneous speed profile of the corresponding shaft, which is explained in detail in the next step. For the ease of estimation of the shaft vibration signal and the reference signal in the subsequent steps, IMF 2 is demodulated to form a normalized sinusoidal function, $\bar{x}_{i m f}(t)$ using the Hilbert transform.

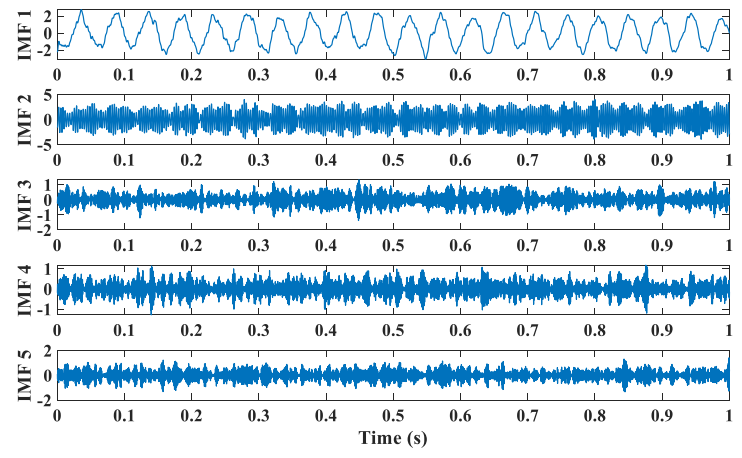

Figure 6. IMFs generated using VMD.

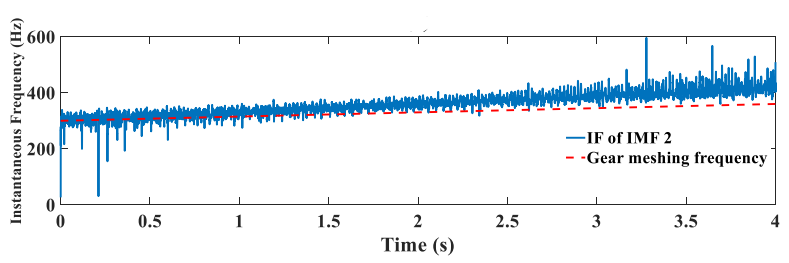

Figure 7. IF of IMF 2 and its comparison with the corresponding gear meshing frequency.

\subsubsection{Step 2: Estimation of the shaft vibration signal}

The next step is to extract a harmonic of the reference shaft rotational frequency $\left(f_{s}(t)\right)$ from the selected IMF (IMF 2), which is a mono-component having IF around the fundamental gear-mesh frequency. Therefore, it can be exploited to extract the first harmonic of $f_{s}(t)$ from where the normalized vibration component of the shaft can be represented as a simple sinusoidal signal as, 


$$
x_{s h}(t)=\cos \left(2 \pi f_{s}(t) t\right) .
$$

Now in order to extract $f_{s}(t)$ from IMF 2, the fixed-axis gearmesh frequency $\left(f_{m}(t)=N_{g} f_{s}(t)\right)$ must be estimated from where the instantaneous speed profile can be calculated as,

$$
f_{s}(t)=\frac{f_{m}(t)}{N_{g}} .
$$

From Eq. (10), it can be observed that the instantaneous phase of the fixed-axis gear's fundamental meshing vibration is given by,

$$
\psi_{g}=2 \pi f_{m}(t) t+b(t)+\varphi .
$$

Now, if we divide Eq. (15) by $2 \pi t$ and consider $b(t) / 2 \pi t$ and $\varphi / 2 \pi t$ to be negligible for an increase in $t$, the gear meshing frequency can be approximated from the instantaneous phase, $\psi_{g}$ of the gear's fundamental mesh vibration signal. Fig. 8 shows the comparison of the estimated instantaneous shaft speed profile and the true instantaneous speed profile, considered for this simulation, and they are found to have a close match.

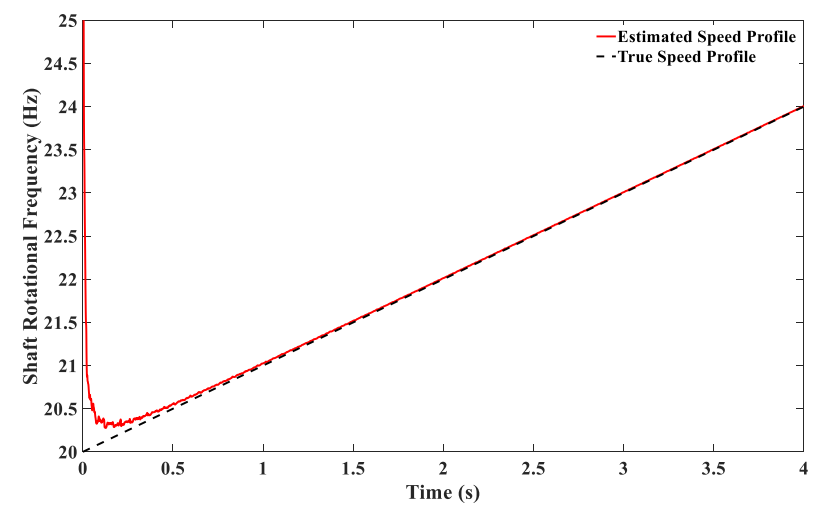

Figure 8. Comparison between the true and the estimated input shaft speed used in the simulation.

\subsubsection{Step 3: Estimation of the reference signal}

In this section, the reference signal, $y(t)$, is built using the estimated vibration signal of the reference shaft of the gearbox. For the reference signal, the gearbox is considered to be operating under a constant load and rotational speed, $f_{s}$. So, the vibrational signal can be written as,

$$
y(t)=A \cos \left(2 \pi \tilde{f}_{s} t\right)
$$

where $A$ is the amplitude. $y(t)$ is constructed using the same sampling frequency and the total time as the original vibration signal $x_{v}(t)$. As in this analysis, the normalized shaft vibration signal is considered, so the amplitude of reference signal, $y(t)$, is set as $A=1$. Furthermore, the constant shaft rotational frequency, $f_{s}$, of the reference signal, $y(t)$, can be chosen from the range between $\frac{p_{p}-1}{T}$ and $\frac{p_{p}+1}{T}$, where $T$ is the total time for which the original vibration signal $x_{v}(t)$ is simulated (Hong et al., 2017). $P_{p}$ corresponds to the number of positive peaks of the sinusoidal signal, which can be estimated from the number of local maximum/minimum points of the normalized shaft-vibration signal.

\subsubsection{Step 4: Fast DTW implementation}

In this step, FDTW is utilized to negate the spectral smearing phenomenon of the measured vibration signal. The two signals, the normalized shaft vibration signal, $x_{s h}(t)$, and the estimated reference signal, $y(t)$, are matched in the time domain by employing the FDTW algorithm. Fig. 9 (a) shows the alignment of $x_{s h}(t)$ and $y(t)$ before warping. The observed phase difference between the two signals is primarily introduced due to speed fluctuation, which is eliminated by aligning $x_{s h}(t)$ with $y(t)$ using FTDW. The resulting warped signals after FDTW are shown in Fig. 9 (b).
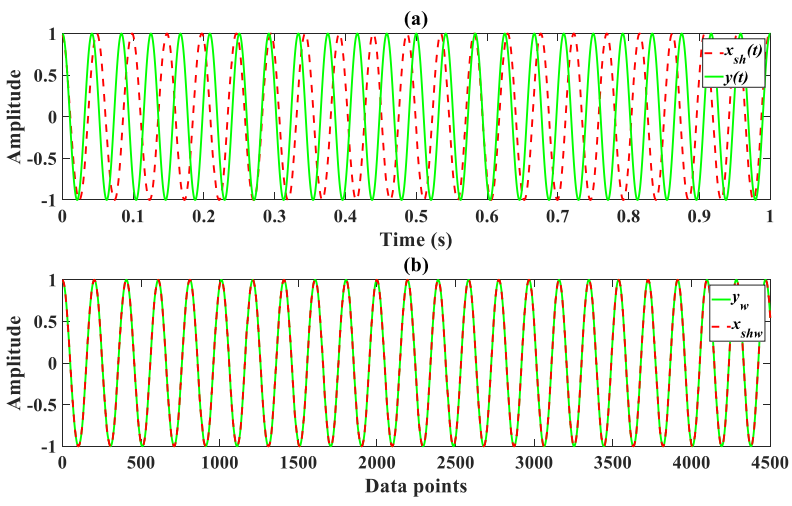

Figure 9. (a) Waveforms of the normalized shaft vibration and the reference signal, and (b) Waveforms of the warped normalized shaft vibration and the warped reference signal.

\subsubsection{Step 5: Reconstruction of the original vibration signal}

After the FDTW step, the original vibration signal, $x_{v}(t)$ is reconstructed based on the data points of warped normalized shaft vibration, $x_{s h w}$. However, it is observed that the length of the reconstructed signal changes and so in order to restore back to the original vibration signal length, a resampling algorithm is applied (Hong et al., 2017). This sequential application of FDTW and resampling algorithm squeezes the time-dependent shaft rotational speed, $f_{s}(t)$, towards the constant rotational speed, $f_{s}$, through optimal alignment of the corresponding similar data points. As a result of this procedure, the time-varying frequency components, which are the harmonics of the shaft rotational speed, $f_{s}(t)$, are also transformed into constant ones. This transformation step makes the proposed detection algorithm suitable for ordertracking analysis, which converts the instantaneous frequency trajectories of specified orders that change 
arbitrarily over time into lines parallel to the time axis on the time-frequency plane. So, the reconstructed vibration signal $\left(x_{w}(t)\right)$ is now used to generate the shaft order spectrum for fault detection.

Fig. 10 shows the generated shaft order spectrum. It can be observed that the severe spectral smearing (Fig. 5) caused by the speed fluctuations is removed from the spectrum and the sidebands introduced due to gear faults can be easily identified. Thus, the proposed algorithm helps in adaptively overcoming the spectral smearing problem in fault detection of gears.

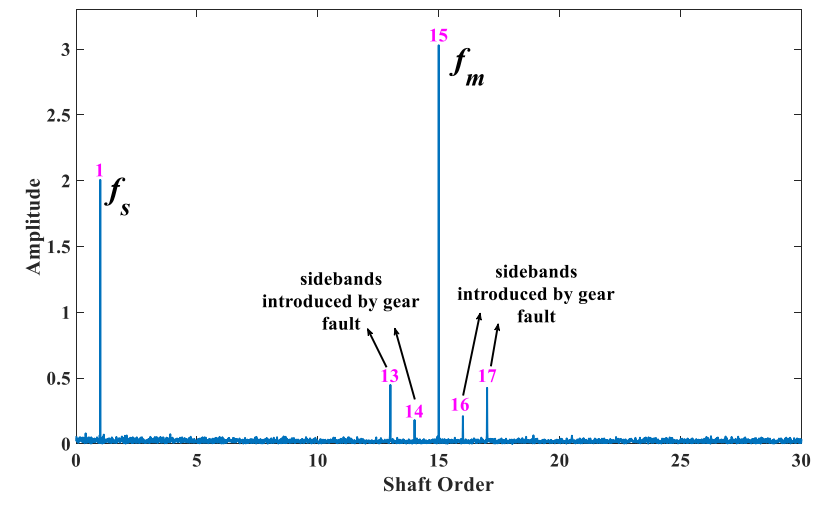

Figure 10. The shaft order spectrum of the reconstructed vibration signal of the gearbox in fault condition in the presence of speed fluctuation and noise after the application of the proposed algorithm.

\section{Conclusion}

In this paper, a self-adaptive fault detection algorithm for gears, combining VMD and FDTW, is presented. Fault detection in industrial gearboxes using measured vibration signal is generally limited by the presence of unwanted background noise and inherent machine operating speed fluctuation. The conventional Fourier transform based approaches suffer from spectral smearing in such cases. The proposed algorithm addresses these issues in order to extract reliable fault information. An analytical simulation model of a fixed-axis gearbox is used to investigate the performance of the proposed detection algorithm. The simulation results indicate that VMD is capable of adaptively extracting a sensitive fault information carrying mode from a noisy gearbox vibration signal, which can then be exploited for order analysis in order to eliminate the smearing effect caused by speed fluctuation. The proposed order-tracking method doesn't require additional speed information of the shaft as the extracted mode is utilized to estimate the shaft speed profile, which is then employed to project the original timescale of the measured vibration signal to a new transformed timescale by performing a warping operation with the help of FDTW. Future work of this study includes implementation of the VMD-FDTW approach in detecting faults using measured vibration signals of practical gearboxes.

\section{ACKNOWLEDGEMENT}

The authors are pleased to acknowledge the University of Auckland for providing the University of Auckland Doctoral Scholarship to Madhurjya D. Choudhury to carry out his doctoral research.

\section{REFERENCES}

Bonnardot, F., El Badaoui, M., Randall, R. B., Daniere, J., \& Guillet, F. (2005). Use of the acceleration signal of a gearbox in order to perform angular resampling (with limited speed fluctuation). Mechanical Systems and Signal Processing, 19 (2005), 766-785.

Cheng, J., Yu, D., Tang, J., \& Yang, Y. (2008). Application of frequency family separation method based upon EMD and local Hilbert energy spectrum method to gear fault diagnosis. Mechanism and Machine Theory, 43 (6), 712 723.

Dragomiretskiy, K., \& Zosso, D. (2014), Variational mode decomposition. IEEE Transactions on Signal Processing, 62 (3), 531-544.

Feng, Z., Zhang, D., \& Zuo, M. J. (2017). Adaptive mode decomposition methods and their applications in signal analysis for machinery fault diagnosis: a review with examples. IEEE Access, 5, 24301-24331.

Hong, L., Qu, Y., Dhupia, J. S., Sheng, S., Tan, Y., \& Zhou, Z. (2017). A novel vibration-based fault diagnostic algorithm for gearboxes under speed fluctuations without rotational speed measurement. Mechanical Systems and Signal Processing, 94 (2017), 14-32.

McFadden, P. D. (1986). Detecting fatigue cracks in gears by amplitude and phase demodulation of the meshing vibration. Journal of Vibration, Acoustics, Stress, and Reliability in Design, 108 (2), 165-170.

Randall, R. B. (1982). A new method of modeling gear faults. Journal of Mechanical Design, 104 (2), 259-267.

Salvador, S., \& Chan, P. (2007). FastDTW: Toward accurate dynamic time warping in linear time and space. Intelligent Data Analysis, 11 (5), 561-580.

Schmidt, S., Heyns, P.S., \& de Villiers, J.P. (2018). A tacholess order tracking methodology based on a probabilistic approach to incorporate angular acceleration information into the maxima tracking process. Mechanical Systems and Signal Processing, 100 (2018), 630-646.

Wang, J., Cheng, F., Qiao, W., \& Qu, L. (2018). Multiscale filtering reconstruction for wind turbine gearbox fault diagnosis under varying-speed and noisy conditions. IEEE Transactions on Industrial Electronics, 65 (5), 4268-4278.

Zosso, D. (2013). Variational mode decomposition. Mathworks.com.https://au.mathworks.com/matlabcentr 
al/fileexchange/44765-variational-mode-

decomposition. (Date accessed 6 April 2019).

\section{BIOGRAPHIES}

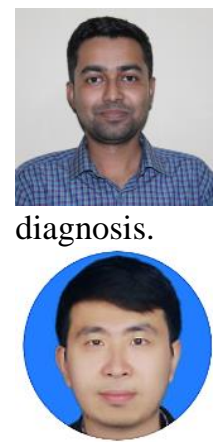

Madhurjya D. Choudhury (M.Tech. IITGuwahati, India, B.Tech. NIT Silchar, India) is currently pursuing his $\mathrm{PhD}$ at the University of Auckland, New Zealand in the field of condition monitoring and fault

Liu Hong (Ph.D. Nanyang Technological University, Singapore, B.E. Huazhong University of Science and Technology, China) is a Lecturer at the Wuhan University of Technology, China. His research interests include fiber optical sensing, and mechanical failure monitoring and diagnosis.

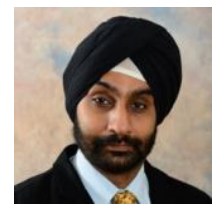

Jaspreet S. Dhupia (Ph.D. \& M.S. University of Michigan, USA, B.Tech. IITDelhi, India) is a senior lecturer at the University of Auckland, New Zealand. Previously he worked as an Assistant Professor at the Nanyang Technological University, Singapore. His research interests include modeling, monitoring and controls of electromechanical drivetrains and power systems. He is the author of more than 60 peerreviewed articles.

Dr. Dhupia is a senior member of the Institute of Electrical and Electronics Engineers (IEEE). He is an Associate Editor for the ASME Dynamics Control and Systems Division. 